\title{
STATUS OF SLIP STACKING AT FERMILAB MAIN INJECTOR*
}

\author{
K. Seiya, T. Berenc, J. Dey, B. Chase, C. Rivetta, I. Kourbanis, J. MacLachlan, K. Meisner, R. \\ Pasquinelli, J. Reid, J. Steimel, Fermilab, Batavia, IL 60510 U.S.A.
}

\begin{abstract}
In order to achieve an increase in proton intensity, the Fermilab Main Injector(MI) will use a stacking process called "slip stacking"[1]. The intensity will be doubled by injecting one train of bunches at a slightly lower energy, another at a slightly higher energy, then bringing them together for the final capture. Beam studies have been performed for this process and we have already verified that, at least for low beam intensities, the stacking procedure works as expected[2]. For high intensity operation, development work of the feedback and feedforward systems was done during the last machine shut down, from August to November 2004[3].
\end{abstract}

\section{INTRODUCTION}

MI accelerates protons and extracts them to a target to produce antiprotons. In the operation cycle, 84 bunches are injected from Booster to MI, accelerated from $8 \mathrm{GeV}$ to $120 \mathrm{GeV}$ and extracted to hit the production target. The total beam intensity was $4.5^{*} 10^{12}$ particles per pulse (ppp) with a cycle of $1.5 \mathrm{sec}$.

Run II upgrade has an intensity goal of $8.0 * 10^{12}$ protons in 84 bunches from the MI and we are currently using a scheme called "slip stacking". On the cycle, 2 bunch trains are injected from Booster and merged to one batch at injection energy of $8 \mathrm{GeV}$, and then accelerated to $120 \mathrm{GeV}$.

On the Run II upgrade, we have to meet three requirements on slip stacking from other machines' operation. First of all, Run II upgrade has a stacking goal of $8.0 * 10^{12}$ protons in 84 bunches from the Main Injector to target. The intensity is going to be almost double the non-slip stacking case. After antiprotons were produced on target, the beam was sent into Debuncher to do bunch rotation and momentum cooling. The bunch length on the target is limited to less than 1.5 nsec because the momentum acceptance of cooling system of Debuncher has a momentum spread of $4 \%$ with the RF voltage of $100 \mathrm{kV}$.[4] MI is providing $120 \mathrm{GeV}$ beam to not only just stacking cycle, but also to fixed target experiment. In order to save time to accelerate beam to fixed target, one additional bunch trains are injected from Booster and MI accelerates third bunch train with slip stacking beam at one cycle. Slip stacking process has to be done before third bunch train comes into MI, so that the time of process limited by two Booster cycle which one cycle is $15 \mathrm{~Hz}$.

We are going to explain the development of the feedback and feedforward systems and operation status in this paper.

\footnotetext{
* Work supported by the Universities Research Assoc., Inc., under
} contract DE-AC02-76CH03000 with the U.S. Dept. of Energy.

\section{BEAM STUDIES WITH LOW INTENSITY}

Fist, beam studies for the slip stacking process have been done with low intensity, $\sim 1.0 * 10^{12} \mathrm{ppp}$, and we have verified the stacking procedure to work as expected.

There was no beam loss during the process, but there was emittance growth when two bunch trains were recaptured. Simulation studies have indicated that the emittance growth is caused by RF phase variation. The RF phase variation was eliminated and the emittance was measured again. No undesirable emittance blow up has been observed. The beam has been accelerated to $120 \mathrm{GeV}$ with a beam loss of $\sim 2 \%$ at the beginning of acceleration.

\section{BEAM LOADING COMPENSATION}

\section{Beam studies}

After beam intensity was increased to $4 * 10^{12}$ ppp, beam loading effects were observed on bunch signal in a mountain range plot as shown in Fig. 1. The signal from the wall current monitor (WCM), reveals the progress of slip stacking from the beginning to the end. The signal was measured with a resolution of $0.5 \mathrm{~ns} / \mathrm{sample}$ and the data were obtained every $2.1 \mathrm{~ms}$ for $0.24 \mathrm{~s}$. There were feedback of $-14 \mathrm{~dB}$ and no feed forward beam loading compensation on Fig. 1, and we saw that particles were outside the RF bucket and the length of train was longer than 84 RF buckets. Feed forward beam loading compensation of $-14 \mathrm{~dB}$ was on the case of Fig. 2 and bunches were keeping the same shape from injection to the end of slip stacking process.

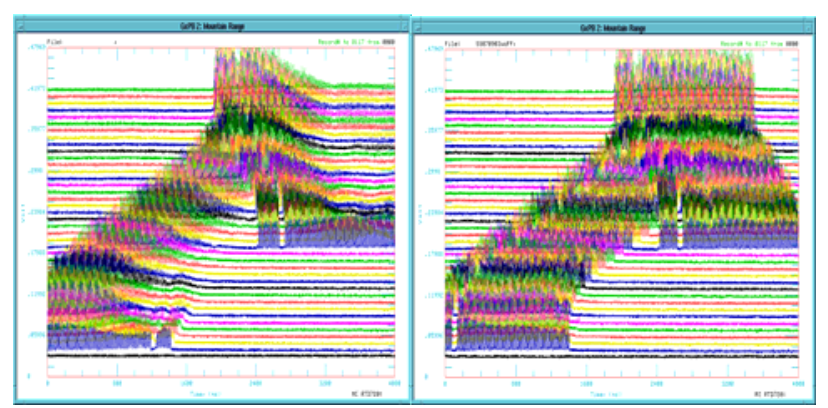

Figure 1 and 2: Mountain range plot with WCM signal. Left and right pictures were with and without feedforward beam loading compensation.

\section{Simulation}

In order to estimate the required gain for beam loading compensation, simulation studies were carried out using the code ESME [5] with beam loading effects. In the simulation studies, two bunch trains of 84 bunches each were put in upper energy and lower energy with a total 
intensity of 1.0E13. The different frequencies were applied to both bunch trains and beam shape was measured after $70 \mathrm{msec}$ as shown in Fig. 3. Some particles were outside the RF bucket and total train length became longer than 84 RF buckets because of the beam loading effects.

Figure 3 shows the result with beam loading compensation in the simulation. Most particles are in the RF bucket and total train length remained to be 84 buckets. In this case feedforward and feedback were applied by $-20 \mathrm{~dB}$ and $-14 \mathrm{~dB}$. The MI RF system currently had $-14 \mathrm{~dB}$ for both. From these results we estimated that MI needs to increase the feedforward compensensation by $-6 \mathrm{~dB}$.

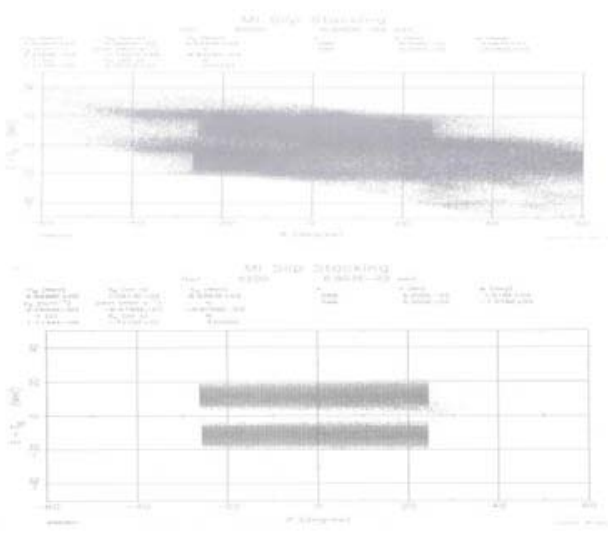

Figure 3 and 4: Simulation results in a phase space. The vertical axis is $40 \mathrm{MeV} / \mathrm{div}$ and horizontal 20 degree/div. Upper was with no beam loading compensation. Lower one was with $-14 \mathrm{~dB}$ feedback and $-20 \mathrm{~dB}$ feedforward beam loading compensation.

\section{RF Stations Upgrades}

In order to have more gains on RF Feed forward loop, RF station has to produce more power of solid state driver. Also PA operation point has to be changed from class $\mathrm{AB}$ to class $\mathrm{A}$ at injection energy so that PA has to be controlled as a function of energy.

During machine shut down, RF stations were upgraded to increased solid state driver from $4 \mathrm{~kW}$ to $8 \mathrm{~kW}$ by adding 4 more modules for each RF station. Programming PA grid became operational as a function of momentum.

Figure 5 shows a frequency spectrum of gap voltage with and without Feed forward compensation on one of the 18 cavities and the gain was $-24 \mathrm{~dB}$. The gain was different on each cavities but average was about $-20 \mathrm{~dB}$.

Figure 6 and 7 show phase detector signal between input and output RF voltage on gap of one RF cavity. The signal was measured from injection to flat top. After we installed full beam loading compensation and PA was programmed, the phase difference was almost 0 degree from injection to flat top.

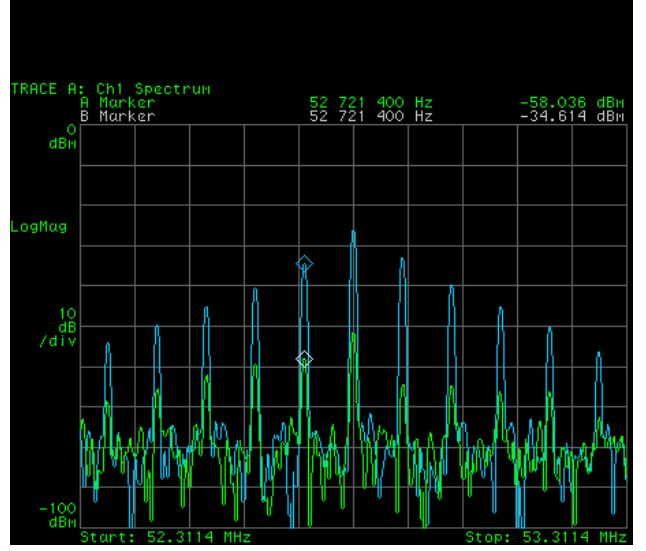

Figure 5: Frequency spectrums of gap voltage monitor on one of the 18 cavities. Blue and green traces show signals without and with feedforward beam loading compensation. The vertical scale is $10 \mathrm{~dB} / \mathrm{div}$.
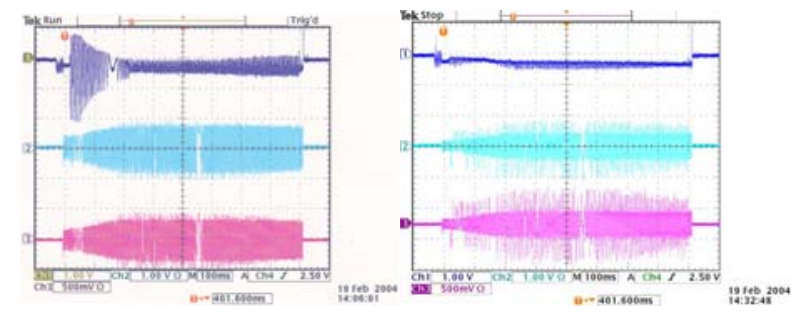

Figure 6 and 7: The top traces (with blue color) are phase detector signal between input and output gap voltage.

\section{OPEARTION STATUS}

Since the end of August in 2005, the Main Injector is operating stacking cycles with slip stacking process. Total intensity injected from Booster is $8.0 * 10^{12} \mathrm{ppp}$ as shown in Fig. 8 and beam on the pbar target is $7.0 * 10^{12} \mathrm{ppp}$. The bunch length at MI extraction is 1.8 nsec. Total time of slip stacking process was $131 \mathrm{msec}$ which was less than two Boostser cycle. We have not achieved our goal of intensity and bunch length, but stacking rate has been improved already. Figure 9 shows the number of pbars as a function of the number of protons on target. After last shut down, the number of pbars was increased by $20 \%$.

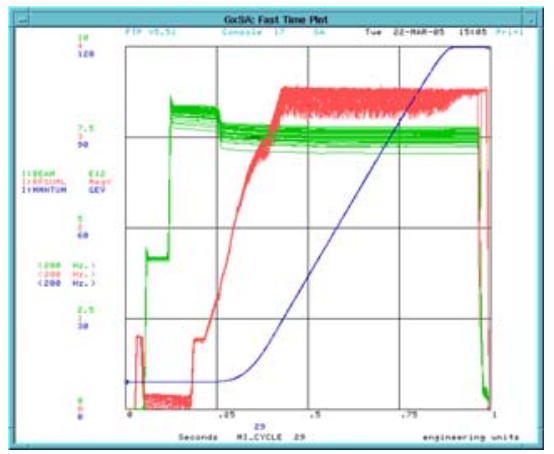

Figure 8: RFSUML: RF voltage[MV], MMNTUM: momentum $[\mathrm{GeV} / \mathrm{c}], \mathrm{I}$ :BEAM: total beam intensity $\left[* 10^{12}\right.$ ppp] 


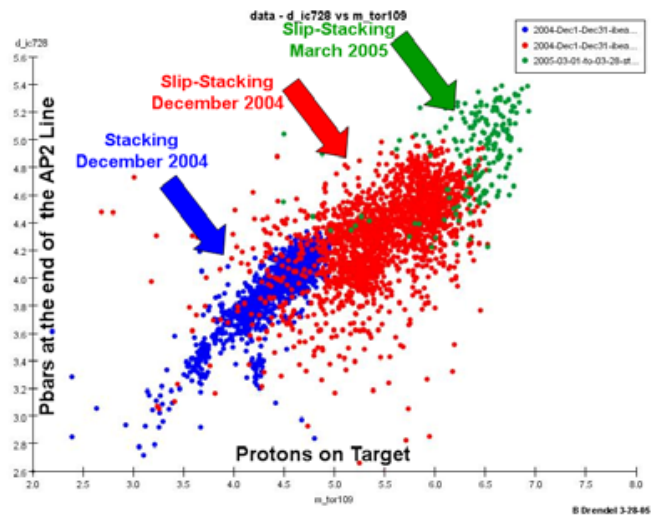

Figure 9: Number of pbars in an ion chamber after the target as a function of the number of protons on target. Blue points are with non slip stacking in December 2004, red is with slip stacking in December 2004, green is is with slip stacking in March 2005.

\section{ISSUES}

We still have beam loss at beginning of acceleration. Intensity at injection $8.0 * 10^{12} \mathrm{ppp}$, at extraction is $7.5^{*} 10^{12} \mathrm{ppp}$ and intensity on pbar target is $7.0^{*} 10^{12} \mathrm{ppp}$. At beginning of acceleration, there was beam loss of $5^{*} 10^{11} \mathrm{ppp}$ and another $5^{*} 10^{11} \mathrm{ppp}$ ppp were sent to MI abort line after stacking beam was sent to target.

\section{Beam loss at injection and leaks from stacking bunches}

Mountain range plots as shown in Fig. 10 and 11 were measured from injection for 47 msec. Fig. 10 and 11 were at the beginning and at the end of bunch train. Some beam started to leak from RF bucket right after injection time on Fig. 10. The direction of the leak depends on beam energy from Booster and the loss never goes away from both side. Longitudinal emittance was measured at MI injection and it was larger than MI bucket area. The leaks were caused by large emittance from Booster and energy mismatch.

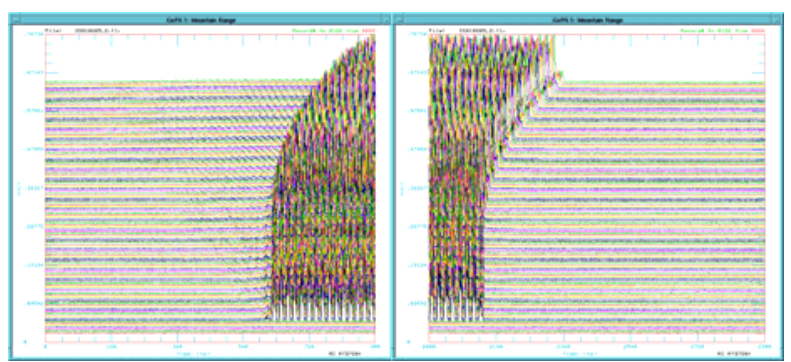

Figure 10 and 11: Mountain range from injection for 47 msec at beginning of bunch train (left) and at the end of bunch train (right).

Since we recaptured the beam with RF voltage of about $1 \mathrm{MV}$ after slipping, and there were leaks around the RF bucket of $90 \mathrm{kV}$, some of the leaking beam was captured with large rf bucket at recapture time and accelerated to $120 \mathrm{GeV}$. Figure 12 shows WCM signal before MI extraction with vertical scale of $300 \mathrm{mV} / \mathrm{div}$ and horizontal scale is 500 nsec. Figure 13 shows WCM signal with vertical scale of $10 \mathrm{mV} / \mathrm{div}$ and the leak is visible outside 84 buckets. After 84 bunches were extracted to target, the left over beam was sent to MI abort. An intensity monitor on the MI abort line shows intensity of 5E11, which agrees with the difference between the intensities of MI extraction and on target.

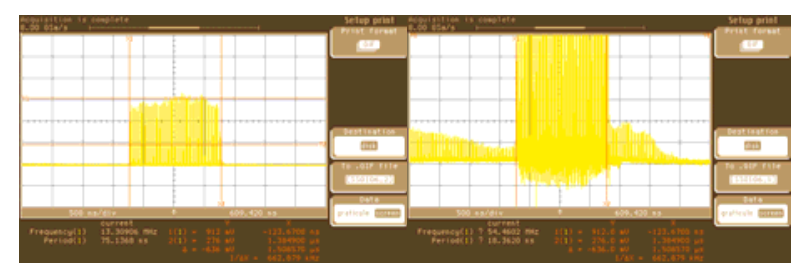

Figure 12 and 13: WCM signal before MI extraction with vertical scale of $300 \mathrm{mV} / \mathrm{div}$ (left) and 10mV/div (right).

\section{Study and operation plans}

In order to get small emittance beam from Booster, beam studies in Booster are on going. Also in MI, collimator will be implemented to get rid of higher momentum beam at injection energy.

\section{CONCLOSTION}

We have done beam studies and simulation studies to estimate that how much feed foreward beam loading compensation is needed for high intensity operation with $8 * 10^{12} \mathrm{ppp}$. The gain of feedforeward system was improved by $-6 \mathrm{~dB}$ with $4 \mathrm{~kW}$ more power on solid state driver. The effects were observed on beam and gap voltage signals.

Since the end of August in 2005, slip stacking is on operation and total intensity on the pbar target is $6.5^{*} 10^{12} \mathrm{ppp}$. The bunch length at MI extraction is 1.8 nsec and total time of slip stacking process was $131 \mathrm{msec}$ which was less than two Boostser cycle. We have increased number of anti protons by $20 \%$ already.

There are beam loss at beginning of acceleration and leaks from stacking bunches at flat top. Intensity at injection $7.5^{*} 10^{12} \mathrm{ppp}$, at extraction is $7.0 * 10^{12} \mathrm{ppp}$ and intensity on pbar target is $6.5^{*} 10^{12} \mathrm{ppp}$. In order to fix those beam loss beam studies are underway.

\section{REFERENCES}

[1] J. A. MacLachlan, "The Beam Dynamics of Slip Stacking”, Fermilab FN-0711, November 2001.

[2] K. Koba, "Slip Stacking experiments at Fermilab Main Injector” ", 2003 PAC, p. 1736, Portland, May 2003.

[3] J. Dey, et al, "53 MHz Beam Loading Compensation for Slip Stacking in the Fermilab Main Injector," PAC '05, These proceedings.

[4] “Plans for TEVATRON Run IIB”, December 2001, p. 106.

[5] J. A. MacLachlan, “Users Guide to ESME”, 2000. 